\title{
Endothelial Cell-Derived Extracellular Vesicles Mitigate Radiation-Induced Hematopoietic Injury
}

\author{
Sadhna O. Piryani, B.S. ${ }^{1,}$, , Yiqun Jiao, M.D. ${ }^{1,{ }^{*}}$, Angel Y.F. Kam, Ph.D. ${ }^{1}$, Yang Liu, Ph.D. ${ }^{2}$, \\ Tuan Vo-Dinh, Ph.D. ${ }^{2}$, Benny J. Chen, M.D. ${ }^{1,3}$, Nelson J. Chao, M.D. ${ }^{1,3}$, Phuong L. Doan, \\ M.D. ${ }^{1,3}{ }^{\wedge}$ \\ ${ }^{1}$ Division of Hematologic Malignancies and Cellular Therapy, Duke University, Durham, NC, USA. \\ ${ }^{2}$ Departments of Biomedical Engineering and Chemistry, Duke University, Durham, NC, USA \\ ${ }^{3}$ Duke Cancer Institute, Duke University, Durham, NC, USA.
}

\section{Abstract}

Purpose.-Extracellular vesicles (EVs) are shed vesicles that bear a combination of nucleic acids and proteins. EVs are becoming recognized as a mode of cell-to-cell communication. Since hematopoietic stem cells (HSCs) reside in proximity to endothelial cells (ECs), we investigated whether EC-derived EVs could regulate HSC regeneration following ionizing radiation.

Methods and Materials.-We generated EVs derived from primary murine marrow ECs. We sought to determine the response of irradiated hematopoietic stem and progenitor cells to syngeneic or allogeneic EVs in culture assays. Starting 24 hours following either sub-lethal or lethal irradiation, mice were treated with EVs or saline or cultured, primary marrow endothelial cells to determine the hematopoietic response in vivo.

Results.-We demonstrate that EVs bear nuclear material and express EC-specific markers. Treatment with EVs promoted cell expansion and increased colony forming units compared to irradiated, hematopoietic cell cultures treated with cytokines alone. Following total body irradiation, EV-treated mice displayed preserved marrow cellularity, marrow vessel integrity, and prolonged overall survival compared to controls treated with saline. Treatment of irradiated HSPCs with EVs from different genetic strains showed similar results to treatment of HSPCs from syngeneic EVs. Mechanistically, treatment of irradiated HSPCs with EVs resulted in decreased

\footnotetext{
^Corresponding Author: Phuong L. Doan, M.D., 905 S. LaSalle Street, DUMC 103866, Durham, NC 27710, Phuong.Doan@duke.edu, Telephone: 919-613-2625, Fax: 919-613-1319.

Author Contributions:

SOP, YJ, AYFK, and PLD performed experiments and analyzed data. SOP, YJ, and PLD developed methodologies. YL and TVD performed measurements of EVs by Nanosight. BJC and PLD provided study supervision. BJC, NJC, and PLD conceived of the study and interpreted data. PLD wrote the manuscript. All authors provided critical review of the manuscript and approved the final manuscript. The authors declare no competing conflicts of interest.

*Equal Contribution.

Authors Responsible for Statistical Analyses:

Sadhna O. Piryani, 905 S. LaSalle Street, DUMC 103866, Durham, NC 27710, 919-613-6632

Angel Y.F. Kam, 905 S. LaSalle Street, DUMC 103866, Durham, NC 27710, 919-684-2691

Phuong L. Doan, as above.

All authors declare no conflicts of interest, except for BJC, NJC, and PLD who are listed as co-inventors on a provisional patent that was filed through the Duke University Office of Licensing and Ventures related to the generation and application of extracellular vesicles described in this manuscript.
} 
levels of annexin V+ apoptotic cell death, which is mediated in part by tissue inhibitor of metalloproteinase-1.

Conclusions.-Our findings show that syngeneic or allogeneic EVs could be cell-derived therapy to deliver physiologic doses of nucleic acids and growth factors to hematopoietic cells in order to accelerate hematopoietic regeneration.

\section{INTRODUCTION}

Hematopoietic stem cells (HSCs) reside in specialized microenvironments that can regulate HSC fate in homeostasis or following injury stress (1). The cells within these specialized microenvironments communicate with HSCs either through direct cell-to-cell contact or secretion of soluble factors (2-5). In the last decade, another mechanism of cell-to-cell communication has been described in that membrane-derived vesicles, or extracellular vesicles (EVs), which bear proteins, lipids, and nucleic acids such as mRNA and non-coding small regulatory microRNAs (miRNAs), could alter the function of either nearby cells or distant cells through transfer of their cargo (6-8). EVs are shed by a variety of cells (i.e., including primary cells and numerous cell lines) and are a heterogeneous population of both exosomes and shed microvesicles that range in size from 50 to $1500 \mathrm{~nm}$ (7-9). EVs can influence normal, physiologic functions such as immune surveillance, coagulation, and maintenance of hematopoietic stem cells (10-12). More recently, EVs that are derived from mesenchymal stem cells (MSC-EVs) have been implicated in tissue repair and regenerative medicine in several pathologic conditions including myocardial ischemia, acute kidney injury (13), fibrotic liver disease (14), neurologic ischemia (15), and hematopoietic regeneration after radiation injury $(16,17)$. The proposed mechanisms by which MSC-EVs augment regeneration were their ability to increase expression of anti-apoptotic genes, downregulate pro-apoptotic genes, and stimulate pro-angiogenic effects by shuttling vascular endothelial growth factor (VEGF), insulin growth factor-1 (IGF-1), and basic fibroblastic growth factor (bFGF) (18). Whether other sources of EVs could contribute to hematopoietic regeneration are unknown.

We sought to determine whether endothelial cell-derived EVs could accelerate hematopoietic stem cell regeneration. Previous studies have demonstrated that transplantation of endothelial cells (ECs) from murine brain, murine fetal blood, or large vessels like aorta could augment hematopoietic regeneration following both lethal and sublethal doses of irradiation (19-22). Surprisingly, transplanted ECs do not engraft in the marrow (21), indicating they could augment hematopoiesis via other mechanisms such as by secreting soluble growth factors like pleiotrophin and epidermal growth factor $(4,23,24)$. Here, using primary sources of marrow ECs to generate EVs, we investigate whether transplantation of these EVs could accelerate hematopoietic regeneration following ionizing radiation. In doing so, we sought to identify whether EVs, along with elaboration of soluble factors, is another mechanism by which ECs promote hematopoietic regeneration. 


\section{METHODS AND MATERIALS}

\section{Animals and Key Biologic Reagents.}

C57BL/6 $\left(\mathrm{H} 2^{\mathrm{b}}, \mathrm{CD} 45.2^{+}\right)$and B6.SJL $\left(\mathrm{H} 2^{\mathrm{b}}, \mathrm{CD} 45.1^{+}\right), \mathrm{BALB} / \mathrm{c}\left(\mathrm{H} 2^{\mathrm{b}}, \mathrm{CD} 45.2^{+}\right)$mice were purchased from Jackson Laboratory (Bar Harbor, ME). Mice were 8 to 12-weeks old at time of experiments. Biologic variables such as age, sex, and weight were matched in all experiments. BALB/c CL.7 murine fibroblast cell line (ATCC TIB-80) was purchased from the Duke Cell Culture Facility. The Duke University Animal Care and Use Committee has approved all animal studies.

\section{Isolation and Characterization of Extracellular Vesicles.}

Primary BM ECs were generated and cultured using previously published methods (4). Conditioned media were isolated using differential ultracentrifugation using previously described methods (25) with slight modifications. For isolation of EVs, media from 7-day culture of EC were collected. EVs were separated by centrifugation at $20^{\circ} \mathrm{C}$ at $300 \mathrm{~g}$ for 15 $\mathrm{min}, 2000 \mathrm{~g}$ for $30 \mathrm{~min}$, and 20,000 $\mathrm{g}$ for $70 \mathrm{~min}$ with collection of the EVs pellet following the last centrifugation. EVs were quantified with Nanosight500 according to manufacturer's instructions (Malvern Panalytical, Inc, Westborough, MA). EVs were used immediately following isolation for all hematopoietic in vitro studies and survival studies. Murine EVs were analyzed by transmission electron microscopy (EM) at the Duke Research Electron Microscopy Core Facility using methods previously published (25). Briefly, EVs were collected in distilled water. Formvar and copper-coated palladium grids were treated with $1 \%$ uranyl acetate solution, $\mathrm{pH} 7$ (Sigma-Aldrich, St. Louis, MO). Following binding of EVs to grids, grids were treated with $1 \%$ uranyl acetate solution, and then images were captured.

\section{Hematopoietic Progenitor Cell Assays and Transplantation Assays.}

Murine BM cells were collected and quantified as previously described (26). Murine BM cells were cultured in murine $20 \mathrm{ng} / \mathrm{ml}$ thrombopoietin, $125 \mathrm{ng} / \mathrm{ml}$ stem cell factor, and 50 ng/ml Flt-3 ligand (TSF, R\&D Systems, Minneapolis, MN). For experiments with C57BL/6 or B6.SJL cells in culture, EVs were supplemented at a range of $5.6 \times 10^{6}-3 \times 10^{13}$ particles per well. For SLAM ${ }^{+} \mathrm{KSL}$ analysis, BM cells were labeled with anti-ckit, antiSca-1, anti-lineage cocktail, anti-CD48, and anti-CD150 antibodies (Biolegend, San Diego, CA; BD, San Jose, CA). For colony forming cells (CFCs) methylcellulose assay, either whole BM or cultured KSL cells and progeny were plated and scored on day 14 according to the manufacturer's protocol (R\&D Systems). Quantification of CFCs was performed independently by two investigators.

For studies with sub-lethal irradiation, mice were irradiated with $500 \mathrm{cGy}$ total body irradiation (TBI), and then injected via tail vein injection of $200 \mu \mathrm{l}$ saline, primary marrow ECs from C57BL/6 mice (passages 3 or earlier), or EVs starting $24 \mathrm{~h}$ after irradiation. For the survival study, C57BL/6 mice were irradiated with $800 \mathrm{cGy}$ TBI. Beginning at $24 \mathrm{~h}$ postirradiation, mice were injected with $200 \mu \mathrm{l}$ saline, primary marrow ECs from C57BL/6 mice (passages 3 or earlier), or EVs. Injections were given at doses of $7.5-11 \times 10^{5}$ cells per dose of ECs or 1.9-4.9 $\times 10^{9}$ particles per dose EVs once daily from days 1 to 4 . Mice were 
sacrificed at specified endpoints or when study endpoints were met according to protocols from Duke IACUC.

Competitive transplantation assays were performed using BM cells from donor B6.SJL mice (CD45.1+). Cells were collected from femurs, stained with CD34 FITC and KSL antibodies as noted above (BD), and isolated by fluorescent-assisted cell sorting on a BD FACSAria II (BD). A thousand CD34-KSL cells were seeded into culture plates for $72 \mathrm{~h}$ with 1 well of donor cells for each recipient mouse. Donor cells and progeny were injected with a competing dose of host $1 \times 10^{5}$ mononuclear cells into C57BL/6 recipient mice that had been lethally-irradiated ( $900 \mathrm{cGy}$ ) $5 \mathrm{~h}$ prior to transplantation. Total CD $45.1^{+}$donor cell engraftment and multi-lineage engraftment were measured in recipient mice every 4 weeks until 16 weeks post-transplantation.

\section{Immunohistochemistry and Cell Survival Assays.}

Femurs were sectioned and stained with mouse endothelial cell antigen (MECA) using methods previously described (4). Femurs were decalcified in Richard-Alien Scientific decalcifying solution (ThermoFisher), dehydrated in a sucrose gradient (5\%-20\%), embedded in OCT media (Sakura Finateck, Torrance, CA). Eight-micrometer sections were cut on a CryoJane Tape System (Instrumedics Inc, Hackensack, NJ), stained with antiMECA antibody (BD) and hematoxylin. Quantification for MECA were performed as previously described using Adobe Photoshop software (20142.2, Adobe Systems, San Jose, CA)(27). Staining of femurs with hematoxylin and eosin staining were performed by the Research Immunohistology Laboratory. Cellular apoptosis and necrosis were analyzed by flow cytometry analysis for annexin V-FITC and 7-aminoactinomycin-D (7-AAD) staining according to manufacturer's specifications (BD).

\section{Protein Array and TIMP-1 analysis.}

Equal volumes of EVs were analyzed using the Milliplex Map Mouse Cytokine/Chemokine Magnetic Bead Panel I and II (Millipore, Burlington, MA). Panel I cytokines were: Eotaxin/ CCL11, G-CSF, GM-CSF, IFN- $\gamma$, IL-1 $\alpha$, IL-1 $\beta$, IL-2, IL-3, IL-4, IL-5, IL-6, IL-7, IL-9, IL-10, IL-12 (p40), IL-12 (p70), IL-13, IL-15, IL-17, IP-10, KC-like, LIF, LIX, MCP-1, MCSF, MIG, MIP-1 $a$, MIP-1 $\beta$, MIP-2, RANTES, TNF- $a$, VEGF. Panel II cytokines were: Erythropoietin (EPO), Exodus-2, Fractalkine/CX3CL1, IFN $\beta 1$, IFN $\gamma$, IL-11, IL-16, IL-17A/F, IL-20, MCP-5/CCL12, MDC (CCL22), MIP-3a/CCL20, MIP-3ß/CCL19, TARC/ CCL17, TIMP-1. Samples were performed in triplicate on Panel I, in duplicate on Panel II, and analyzed on a BioPlex 200 System (Biorad, Hercules, CA). Data were normalized to baseline levels of protein contained in culture media. Sample analyses were performed at the Duke Regional Biocontainment Laboratory (RBL).

For TIMP-1 analysis, RNA was extracted using RNeasy mini-kit (Qiagen, Germantown, MD), complementary DNA was generated with High capacity cDNA Reverse Transcription kit, and real-time PCR for TIMP-1 detection was performed with Taqman Primer according the manufacturer's specifications (ThermoFischer). Data for RTPCR were normalized to GAPDH and are shown following $\Delta \Delta \mathrm{C}_{\mathrm{T}}$ analysis (28). C57BL/6 bone marrow lineagenegative cells were isolated with MACS microbeads and LS columns according to the 
manufacturer specifications (Miltenyi Biotech). Following culture with recombinant murine TIMP-1 (R\&D Systems), cells were collected and analyzed by flow cytometric analysis for cell survival as described above.

\section{Statistical Analyses and Image Capture.}

Data are shown as means \pm SEM. Analyses were performed with GraphPad Prism (v7.0, La Jolla, CA) using either Student's t test (two-tailed with unequal variance) or Log-rank test for survival analysis. Images for transmission electron microscopy were obtained on a Philips EM300 electron microscope (FEI-Philips, Hillsboro, OR) with AMTV 600 software (Advanced Microscopy Techniques, Danvers, MA). Images for immunohistochemistical analysis were obtained with Zeiss Axiolmager Z2 and Axiocam 506 (San Diego, CA).

\section{RESULTS}

Since primary murine marrow EVs have not been previously generated, we sought to isolate and characterize these EVs. As described in detail in the Methods section, EVs were isolated from primary BM EC cultures. Transmission electron microscopy and nanoparticle light scattering tracking analysis of these EVs demonstrate rounded vesicles with most EVs are less than $200 \mathrm{~nm}$ in size (Fig. 1A, B). To further characterize that these EVs are shed from ECs, we used real-time PCR to demonstrate that EVs express both CD31 and VECadherin, which are highly enriched in endothelial cells (29-31), and do not express fibroblast-specific protein 1 (FSP-1), a marker for fibroblasts (32) (Fig. 1C). Conversely, a murine fibroblast cell line BALB/c CL.7 strongly expresses FSP-1, while CD31 and VECadherin are not detected (Fig. 1C). These data demonstrate that EVs are shed from ECs in culture and bear a phenotype consistent with ECs.

Without radiation injury, cultures of HSPCs and EVs resulted in no differences in cell expansion or capacity to generate colony-forming units (CFCs, Fig. S1). To determine whether EVs could mitigate hematopoietic injury following ionizing irradiation, irradiated C57BL/6 hematopoietic stem/progenitor cells (HSPCs, ckit+Sca+Lin-, KSL) cells were isolated and cultured with EVs or cytokines alone (thrombopoietin, stem cell factor, and Flt-3 ligand, TSF). At 7 days in culture following $300 \mathrm{cGy}$, KSL cells with EVs demonstrate a 5-fold increase in total cells and an increase in percentage KSL cells (Fig. 2A, B). In cultures with a more primitive stem cell population of CD34-KSL cells, EVs could also increase expansion of irradiated cells and provide a 2-fold increase in colony-forming units compared to TSF alone (Fig. 2C). Using competitive transplantation assays to measure longterm HSC content (LT-HSC) (33), irradiated CD34-KSL cells treated with EVs did not increase HSC content compared to cultures with TSF alone (Fig. S2). These data demonstrate the EVs could expand HSPC content following irradiation, possibly by promoting differentiation of part of the stem cell pool.

Since these EVs are generated from primary marrow ECs and could have biologic, batch-tobatch variability, we sought to determine whether there exists a linear dose-response for treatment of EVs. Following irradiation and 7-day culture of KSL cells with increasing doses of EVs (range 0 to $16.8 \times 10^{9}$ particles per well), KSL cells expanded in a near linear manner, with maximum cell expansion at a concentration of $10 \times 10^{9}$ particles per well (Fig. 
2D). In cultures of irradiated CD34-KSL cells, maximum cell expansion was achieved with $2.5 \times 10^{9}$ particles per well. These data suggest that irradiated HSPCs respond to EVs in a dose-dependent manner. Moreover, these comparisons indicate that CD34-KSL cells may be more sensitive to EVs compared to KSL cells since fewer particles are needed to achieve maximum cell expansion.

To determine whether EVs could promote hematopoietic regeneration in vivo, we measured hematopoietic cell function and overall survival in irradiated mice treated with either EVs or saline and compared these results to EC-treated animals. At day 7 following $500 \mathrm{cGy}$ total body irradiation (TBI), which is a myelosuppressive dose, we noted increased marrow cellularity and vessel integrity in the EVs- and EC-treated mice compared to saline-treated mice (Fig. 3 A-C). Both percentage KSL and SLAM+KSL (CD150+CD48-KSL) were increased in EVs- and EC-treated mice compared to saline-treated mice (Fig. 3D-F). This increase in HSPC content was associated with increased CFC content as well (Fig. 3G). Importantly, there were no differences in HSPC content or function between EVs-and ECtreated groups, indicating that EVs and ECs could comparably promote hematopoietic regeneration. When mice are exposed to lethal dose irradiation ( $800 \mathrm{cGy}$ ), saline-treated mice had a median survival of 16 days and zero of 10 mice survived until day 30 (Fig. 3H, I). In contrast, $50 \%$ (5 of 10 animals) and 70\% (7 of 10 animals) survived more than 30 days in the EVs- and EC-treated groups, respectively. There were no differences in survival when comparing EC to EVs-treated animals, indicating that EVs were comparably effective as EC at prolonging survival following lethal-dose irradiation (Fig. 3H, I).

Since EVs expand irradiated HSPCs both in vitro and in vivo, we next sought to determine whether EVs generated from primary marrow ECs in mice of different genetic strains could replicate these results (Fig. 4A, B). When HSPC cultures were treated with an equal number of particles of EVs that have been generated from either C57BL/6 ECs (syngeneic) or BALB/c ECs (allogeneic), both EVs expanded irradiated, C57BL/6 HSPCs and increased CFCs compared to TSF cultures (Fig. 4C). There were no differences between the groups of EVs for either cell counts or CFCs. To test this in hematopoietic cells from a different genetic strain, irradiated BALB/c HSPCs were cultured with C57BL/6 EVs, BALB/c EVs, or TSF alone. Again, similar results were confirmed that EVs could mitigate radiation injury to HSPCs (Fig. 4D). Next, we asked whether allogeneic transplantation of EVs could mitigate hematopoietic radiation injury in vivo. Irradiated BALB/c mice were transplanted with C57BL/6 EVs starting $24 \mathrm{~h}$ after $500 \mathrm{cGy}$ TBI (Fig. 4E). At day 7, EV-treated mice display increased marrow cellularity compared to saline-treated mice (Fig. 4F). Hematopoietic stem/progenitor cell function, as measured by CFCs, was increased in EVtreated mice compared to saline-treated mice (Fig. 4G). Since no differences were observed between the groups of EVs for either genetic stain, these data suggest that EVs may be manufactured using universal donors.

Since ECs mitigate acute radiation injury to HSCs in part by attenuating apoptotic cell death, we measured the impact of EV treatment on HSPCs. EVs decreased annexin V+ cells from irradiated HSPC cultures compared to cultures with TSF alone (Fig. 5A). To further examine how EVs decrease apoptotic cell death and characterize the content of EVs, we screened several known hematopoietic growth factors and discovered that EVs contain stem cell 
factor (SCF), but not granulocyte colony-stimulating factor (G-CSF) or granulocytemacrophage colony-stimulating factor (GM-CSF, Fig. S3). Moreover, we performed an array of 48 cytokines and chemokines and found that tissue inhibitor of metalloproteinase 1 (TIMP-1) was most abundantly expressed in EVs compared to other measured cytokines (Fig. 5B). TIMP-1 was 10-fold higher than the next highest expressing cytokine monocyte chemoattractant protein-1b (MCP-1b) (Fig. 5B). Using real-time PCR, we assessed mRNA content of EVs and confirmed that TIMP-1 mRNA was more abundantly expressed in EVs compared to fibroblasts, and not as abundant as ECs (Fig. 5C). While irradiated HSPCs cultured with TIMP-1 displayed no differences in total cell counts of \% KSL cells (Fig. S4), the levels of annexin V+ cells were decreased compared to cultures with TSF alone (Fig. 5D). These data demonstrate that EVs mediate improved hematopoietic cell survival following irradiation at least in part by TIMP-1.

\section{DISCUSSION}

We have developed methods to both generate and quantify EVs from primary marrow ECs. Historically, primary ECs, especially marrow ECs, were difficult to culture and propagate, with cells exhausting following only a few passages. The use of primary EC-derived EVs will more fully recapitulate hematopoietic regeneration compared to EC-derived EVs that are derived from cell lines.

We demonstrate that EC-derived EVs could accelerate hematopoietic regeneration following radiation injury using both in vitro and in vivo systems. While EVs did not increase LT-HSC content as measured by competitive transplantation assays, EVs increased HSPC content and was sufficient to prolong survival after irradiation. It is possible that higher doses of EVs than the ones used in these experiments are required to measure a difference in HSC content by competitive transplantation. Alternatively, since EVs promoted marked expansion of HSPC compared to cultures with cytokines alone, EVs could induce differentiation of HSPCs and thus decrease HSC content.

EVs may have better efficacy compared to U.S Food and Drug Administration (FDA)approved drugs for mitigation of the acute hematopoietic syndrome. The FDA has approved two drugs, G-CSF and its PEGylated version for the treatment of neutropenia associated with acute radiation syndrome. In a recent study to examine the optimal dosing strategy of G-CSF, three doses of G-CSF starting $24 \mathrm{~h}$ following radiation resulted in an overall survival advantage of $30 \%$ compared to control animals (34). When 4 doses of EVs are administered starting $24 \mathrm{~h}$ following radiation, we achieve an overall survival advantage of 50\% compared to animals that receive saline control injections. These data indicate that EVs are at least comparable and likely superior to G-CSF for prolonging survival after lethal-dose irradiation. Since EVs do not express G-CSF or GM-CSF, EVs and G-CSF likely work through different pathways, which raises the possibility that EVs and G-CSF could work synergistically.

We identified that EVs bear high levels of TIMP-1. This finding is consistent with a previously published report that EVs from human umbilical vein ECs (HUVECs) contain high levels of TIMP-1 (35). Interestingly, mice that are deficient in TIMP-1 display aberrant 
microvascular networks, deranged vascular integrity, and diminished vascular remodeling following ischemia (36). Conversely, increased levels of TIMP-1 is associated with poorer prognosis in colorectal cancer with increased liver metastasis (37) and also promoted angiogenesis in a model of lung adenocarcinoma (38). Yet, no reports have associated TIMP-1 with regenerative potential. Here, we show that treatment of irradiated HSPCs with TIMP-1 was sufficient to ameliorate radiation-induced cell death. Whether there exists synergistic activity between TIMP-1, other members of TIMP family, and other described hematopoietic growth factors (i.e., EGF, VEGF, cytokines from protein array) are not yet known.

The use of EVs to deliver therapeutic doses of nucleic acids, proteins, and other cargo following radiation injury could have potential benefits over transplantation of cellular products (i.e., hematopoietic cells, endothelial cells). In certain organ systems, like pulmonary or liver, transplanted ECs will home and directly engraft to mediate organ repair following partial organ resection $(39,40)$. In bone marrow, however, transplanted ECs do not engraft, and so EVs could provide a therapeutic advantage by exerting its effect either through direct activity on hematopoietic cells or via paracrine signaling through its cargo of cytokines and nucleic acids. Since EVs shuttle complex biologic information, EC-tohematopoietic cell communication via EVs may provide a more physiologic response to radiation injury compared to single growth factors to mediate a hematopoietic response. Since EVs do not appear to be immunologically active, EVs may be developed for off-theshelf administration without the need for stringent HLA-matching and prolonged immunosuppressive agents.

\title{
CONCLUSIONS
}

EVs could be an optimal source of cellular therapy following radiation-induced hematopoietic injury or other organ damage.

\section{Supplementary Material}

Refer to Web version on PubMed Central for supplementary material.

\section{Source of Financial Support/Funding Statement/Acknowledgements:}

\begin{abstract}
Biomarker profiling was performed under the management of Dr. Andrew N. Macintyre and direction of Dr. Gregory D. Sempowski in the Immunology Unit of the Duke Regional Biocontainment Laboratory (RBL), which received partial support for construction from the National Institutes of Health, National Institute of Allergy and Infectious Diseases (UC6-AI058607). This work was supported by the Opportunity Funds Management Core of the Centers for Medical Countermeasures against Radiation, National Institute of Allergy and Infectious Diseases; grant number U19AI067773 (BJC and PLD), the National Cancer Institute of the National Institutes of Health under Award Number K08CA184552 (PLD), the Duke Strong Start Scholars Program (PLD), and the Duke Cancer Institute (PLD).
\end{abstract}

\section{REFERENCES}

1. Mendelson A, and Frenette PS. Hematopoietic stem cell niche maintenance during homeostasis and regeneration. Nat Med 2014;20:833-46. [PubMed: 25100529]

2. Doan PL, and Chute JP. The vascular niche: Home for normal and malignant hematopoietic stem cells. Leukemia 2012;26:54-62. [PubMed: 21886170] 
3. Ding L, and Morrison SJ. Haematopoietic stem cells and early lymphoid progenitors occupy distinct bone marrow niches. Nature 2013;495:231-5. [PubMed: 23434755]

4. Doan PL, Himburg HA, Helms K, Russell JL, Fixsen E, Quarmyne M, et al. Epidermal growth factor regulates hematopoietic regeneration after radiation injury. Nat Med 2013;19:295-304. [PubMed: 23377280]

5. Himburg HA, Doan PL, Quarmyne M, Yan X, Sasine J, Zhao L, et al. Dickkopf-1 promotes hematopoietic regeneration via direct and niche-mediated mechanisms. Nat Med 2017;23:91-9. [PubMed: 27918563]

6. Lee Y, El Andaloussi S, and Wood MJ. Exosomes and microvesicles: Extracellular vesicles for genetic information transfer and gene therapy. Hum Mol Genet 2012;21:R125-34. [PubMed: 22872698]

7. Xu R, Greening DW, Zhu HJ, Takahashi N, and Simpson RJ. Extracellular vesicle isolation and characterization: Toward clinical application. J Clin Invest 2016;126:1152-62. [PubMed: 27035807]

8. Pitt JM, Kroemer G, and Zitvogel L. Extracellular vesicles: Masters of intercellular communication and potential clinical interventions. J Clin Invest 2016;126:1139-43. [PubMed: 27035805]

9. Flamant S, and Tamarat R. Extracellular vesicles and vascular injury: New insights for radiation exposure. Radiat Res 2016;186:203-18. [PubMed: 27459703]

10. Raposo G, Nijman HW, Stoorvogel W, Liejendekker R, Harding CV, Melief CJ, et al. B lymphocytes secrete antigen-presenting vesicles. J Exp Med 1996;183:1161-72. [PubMed: 8642258]

11. Del Conde I, Shrimpton CN, Thiagarajan P, and Lopez JA. Tissue-factor-bearing microvesicles arise from lipid rafts and fuse with activated platelets to initiate coagulation. Blood 2005;106:1604-11. [PubMed: 15741221]

12. Ratajczak J, Miekus K, Kucia M, Zhang J, Reca R, Dvorak P, et al. Embryonic stem cell-derived microvesicles reprogram hematopoietic progenitors: Evidence for horizontal transfer of mrna and protein delivery. Leukemia 2006;20:847-56. [PubMed: 16453000]

13. Bruno S, Grange C, Collino F, Deregibus MC, Cantaluppi V, Biancone L, et al. Microvesicles derived from mesenchymal stem cells enhance survival in a lethal model of acute kidney injury. PLoS One 2012;7:e33115. [PubMed: 22431999]

14. Lou G, Yang Y, Liu F, Ye B, Chen Z, Zheng M, et al. Mir-122 modification enhances the therapeutic efficacy of adipose tissue-derived mesenchymal stem cells against liver fibrosis. J Cell Mol Med 2017;21:2963-73. [PubMed: 28544786]

15. Xin H, Li Y, Liu Z, Wang X, Shang X, Cui Y, et al. Mir-133b promotes neural plasticity and functional recovery after treatment of stroke with multipotent mesenchymal stromal cells in rats via transfer of exosome-enriched extracellular particles. Stem Cells 2013;31:2737-46. [PubMed: 23630198]

16. Wen S, Dooner M, Cheng Y, Papa E, Del Tatto M, Pereira M, et al. Mesenchymal stromal cellderived extracellular vesicles rescue radiation damage to murine marrow hematopoietic cells. Leukemia 2016;30:2221-31. [PubMed: 27150009]

17. Schoefinius JS, Brunswig-Spickenheier B, Speiseder T, Krebs S, Just U, and Lange C. Mesenchymal stromal cell-derived extracellular vesicles provide long-term survival after total body irradiation without additional hematopoietic stem cell support. Stem Cells 2017;35:2379-89. [PubMed: 29024236]

18. Choi HY, Moon SJ, Ratliff BB, Ahn SH, Jung A, Lee M, et al. Microparticles from kidney-derived mesenchymal stem cells act as carriers of proangiogenic signals and contribute to recovery from acute kidney injury. PLoS One 2014;9:e87853. [PubMed: 24504266]

19. Montfort MJ, Olivares CR, Mulcahy JM, and Fleming WH. Adult blood vessels restore host hematopoiesis following lethal irradiation. Exp Hematol 2002;30:950-6. [PubMed: 12160847]

20. Chute JP, Muramoto GG, Salter AB, Meadows SK, Rickman DW, Chen B, et al. Transplantation of vascular endothelial cells mediates the hematopoietic recovery and survival of lethally irradiated mice. Blood 2007;109:2365-72. [PubMed: 17095624]

21. Salter AB, Meadows SK, Muramoto GG, Himburg H, Doan P, Daher P, et al. Endothelial progenitor cell infusion induces hematopoietic stem cell reconstitution in vivo. Blood 2009;113:2104-7. [PubMed: 19141867] 
22. Zachman DK, Leon RP, Das P, Goldman DC, Hamlin KL, Guha C, et al. Endothelial cells mitigate DNA damage and promote the regeneration of hematopoietic stem cells after radiation injury. Stem Cell Res 2013;11:1013-21. [PubMed: 23939266]

23. Chute JP, Muramoto GG, Fung J, and Oxford C. Soluble factors elaborated by human brain endothelial cells induce the concomitant expansion of purified human bm cd34+cd38- cells and scid-repopulating cells. Blood 2005;105:576-83. [PubMed: 15345596]

24. Himburg HA, Muramoto GG, Daher P, Meadows SK, Russell JL, Doan P, et al. Pleiotrophin regulates the expansion and regeneration of hematopoietic stem cells. Nat Med 2010;16:475-82. [PubMed: 20305662]

25. Thery C, Amigorena S, Raposo G, and Clayton A. Isolation and characterization of exosomes from cell culture supernatants and biological fluids. Curr Protoc Cell Biol 2006; Chapter 3:Unit 322.

26. Piryani SO, Kam AYF, Kliassov EG, Chen BJ, Spector NL, Chute JP, et al. Epidermal growth factor and granulocyte colony stimulating factor signaling are synergistic for hematopoietic regeneration. Stem Cells 2018;36:252-64. [PubMed: 29086459]

27. Lehr HA, Mankoff DA, Corwin D, Santeusanio G, and Gown AM. Application of photoshop-based image analysis to quantification of hormone receptor expression in breast cancer. J Histochem Cytochem 1997;45:1559-65. [PubMed: 9358857]

28. Livak KJ, and Schmittgen TD. Analysis of relative gene expression data using real-time quantitative pcr and the 2(-delta delta $c(t))$ method. Methods 2001;25:402-8. [PubMed: 11846609]

29. Newman PJ. The biology of pecam-1. J Clin Invest 1997;99:3-8. [PubMed: 9011572]

30. Avecilla ST, Hattori K, Heissig B, Tejada R, Liao F, Shido K, et al. Chemokine-mediated interaction of hematopoietic progenitors with the bone marrow vascular niche is required for thrombopoiesis. Nature Medicine 2004;10:64-71.

31. May C, Doody JF, Abdullah R, Balderes P, Xu X, Chen CP, et al. Identification of a transiently exposed ve-cadherin epitope that allows for specific targeting of an antibody to the tumor neovasculature. Blood 2005;105:4337-44. [PubMed: 15701713]

32. Strutz F, Okada H, Lo CW, Danoff T, Carone RL, Tomaszewski JE, et al. Identification and characterization of a fibroblast marker: Fsp1. J Cell Biol 1995;130:393-405. [PubMed: 7615639]

33. Purton LE, and Scadden DT. Limiting factors in murine hematopoietic stem cell assays. Cell Stem Cell 2007;1:263-70. [PubMed: 18371361]

34. Satyamitra M, Kumar VP, Biswas S, Cary L, Dickson L, Venkataraman S, et al. Impact of abbreviated filgrastim schedule on survival and hematopoietic recovery after irradiation in four mouse strains with different radiosensitivity. Radiat Res 2017;187:659-71. [PubMed: 28362168]

35. Taraboletti G, D’Ascenzo S, Borsotti P, Giavazzi R, Pavan A, and Dolo V. Shedding of the matrix metalloproteinases mmp-2, mmp-9, and $\mathrm{mt} 1-\mathrm{mmp}$ as membrane vesicle-associated components by endothelial cells. Am J Pathol 2002;160:673-80. [PubMed: 11839588]

36. Mandel ER, Uchida C, Nwadozi E, Makki A, and Haas TL. Tissue inhibitor of metalloproteinase 1 influences vascular adaptations to chronic alterations in blood flow. J Cell Physiol 2017;232:83141. [PubMed: 27430487]

37. Illemann M, Eefsen RH, Bird NC, Majeed A, Osterlind K, Laerum OD, et al. Tissue inhibitor of matrix metalloproteinase-1 expression in colorectal cancer liver metastases is associated with vascular structures. Mol Carcinog 2016;55:193-208. [PubMed: 25594187]

38. Rojiani MV, Ghoshal-Gupta S, Kutiyanawalla A, Mathur S, and Rojiani AM. Timp-1 overexpression in lung carcinoma enhances tumor kinetics and angiogenesis in brain metastasis. $\mathrm{J}$ Neuropathol Exp Neurol 2015;74:293-304. [PubMed: 25756591]

39. Ding BS, Nolan DJ, Butler JM, James D, Babazadeh AO, Rosenwaks Z, et al. Inductive angiocrine signals from sinusoidal endothelium are required for liver regeneration. Nature 2010;468:310-5. [PubMed: 21068842]

40. Rafii S, Ginsberg M, Scandura J, Butler JM, and Ding BS. Transplantation of endothelial cells to mitigate acute and chronic radiation injury to vital organs. Radiat Res 2016;186:196-202.

[PubMed: 27459700] 
A

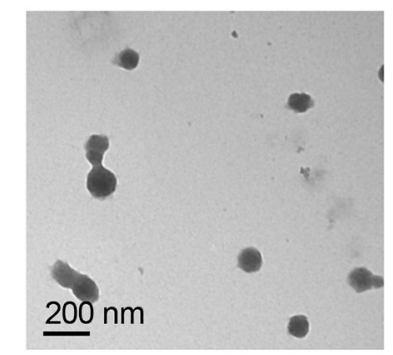

B

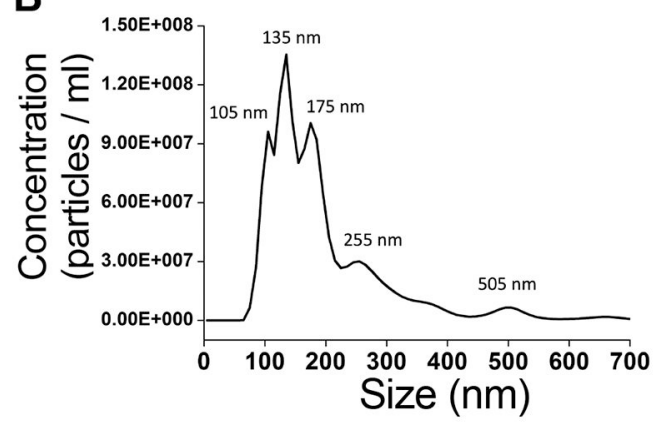

C
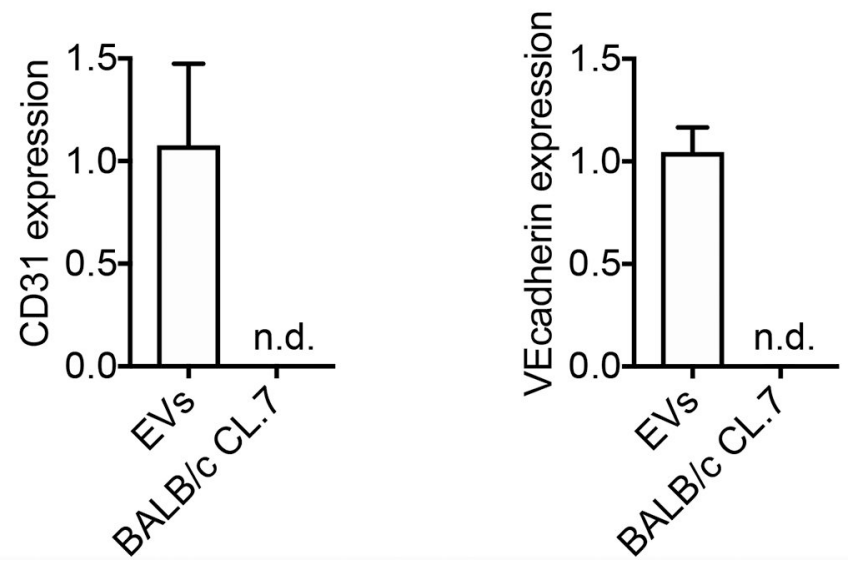
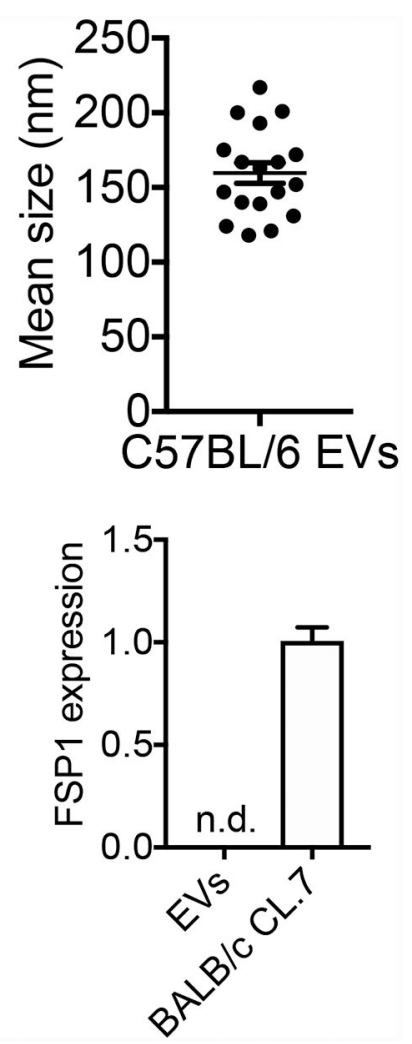

Figure 1. Characterization of endothelial cell-derived EVs.

(A) Transmission electron microscopy image of EVs generated from C57BL/6 marrow ECs. Scale bar $200 \mathrm{~nm}$. (B) Left, size distribution profiles for EVs generated from C57BL/6 EC as measured by Nanosight500. Right, mean size of EVs. Mean size is $160 \mathrm{~nm}$. $n=18$. (C) Levels of mRNA expression of CD31, VEcadherin, and FSP1 in C57BL/6 EVs or BALB/c CL.7 cells. Data are shown as means \pm SEM. n.d., not detected. 
A

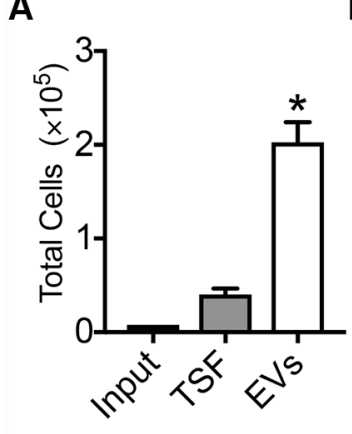

C

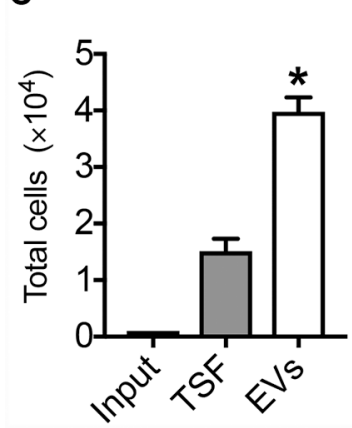

B

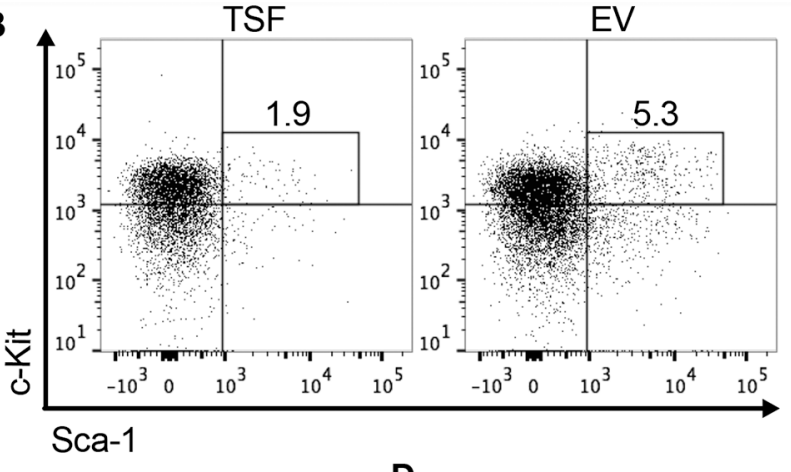

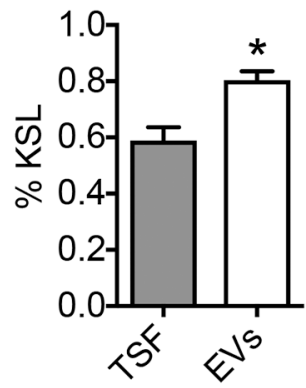
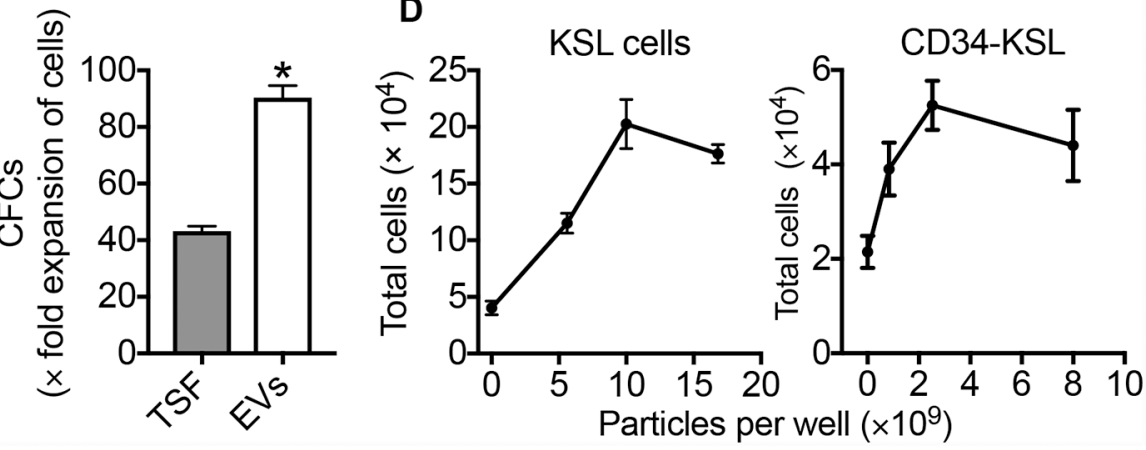

Figure 2. EVs expand irradiated, hematopoietic stem/progenitor cells in vitro. C57BL/6 KSL cells were irradiated (300 cGy) and then cultured for 7 days with TSF or TSF + EVs. (A) Total cells, (B) FACS analysis, and quantification of percentage KSL cells shown following gating of Lin- cells. $n=3-6$ per group. ${ }^{*} p<0.0001$ and 0.01 for total cells and $\%$ KSL, respectively. (C) Total cells and CFCs of irradiated (300 cGy) CD34 KSL cells at day 7 in culture with TSF or TSF + EVs. $n=6-9$ per group. $* p<0.0001$ for both total cells and CFCs. Input was $10^{4}$ cells per dish for culture of CFCs. (D) Dose response curves of total cells to number of EV particles per well from irradiated (300 cGy) KSL cells (left) or CD34KSL cells (right) following 7-day culture. Input was 7,500 or 1,000 cells for KSL and CD34-KSL, respectively. Data are shown as means \pm SEM. Student's t test (two-tailed with unequal variance) was applied to these data. 


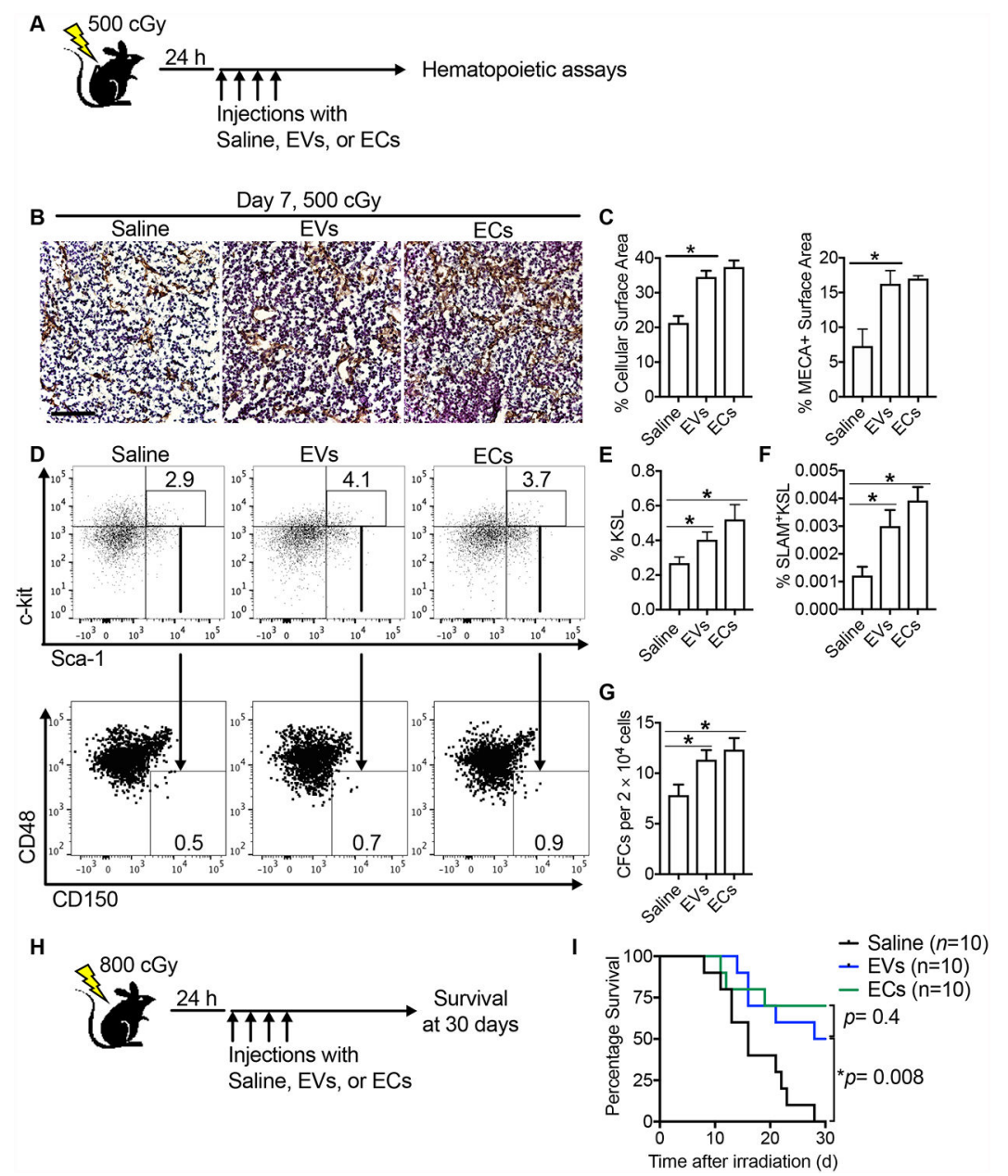

Figure 3. EVs mitigate ionizing radiation injury to hematopoietic stem and progenitor cells. (A) Schematic diagram of study design. C57BL/6 mice were treated with $500 \mathrm{cGy}$ total body irradiation (TBI), and then starting $24 \mathrm{~h}$ later, were injected with saline, endothelial cell-derived extracellular vesicles (EVs), or primary marrow endothelial cells (ECs) from day 1-4. (B) Femurs stained with mouse endothelial cell antigen (MECA, brown) and hematoxylin display increased cellularity and preserved BM EC architecture in mice treated with EVs and ECs compared to saline-treated mice. Scale bar $100 \mu \mathrm{m}$. (C) Left, Quantification of cellularity. $n=2-4$ per group. * $p=0.003$. Right, Quantification of MECA +staining. $n=2-4$ per group, ${ }^{*} p=0.03$. (D) Representative flow cytometry analysis for KSL and SLAM+KSL. Gating is from Lin- cell population. (E) Quantification of percentage KSL. $n=5$ per group, ${ }^{*} p=0.04$ and 0.03 for Saline vs. EVs and ECs, respectively. $p=0.3$ for EVs vs. ECs. (F) Quantification of percentage SLAML+KSL. $n=5$ per group, ${ }^{*} p=0.03$ and 0.002 for Saline vs. EVs and ECs, respectively. (G) Levels of CFCs, $n=6$ per group. * $p=$ 0.03 and 0.02 for Saline vs. EVs and ECs, respectively. $p=0.5$ for EVs vs. ECs. Data are shown as means \pm SEM. Student's t test (two-tailed with unequal variance) was applied to these data. (H) Schematic diagram of study design. C57BL/6 mice were irradiated with 800 cGy TBI. Starting $24 \mathrm{~h}$ later, mice were injected once daily on days 1-4 via tail vein with saline (black line), EVs (blue line), or primary marrow ECs (green line). (I) Analysis of 
survival at day 30. $n=10$ mice per group. ${ }^{*} p=0.008$ and 0.004 for Saline vs. EVs and EC, respectively. $p=0.5$ for EVs vs. EC. Survival analysis as performed using Log-rank analysis. 
A

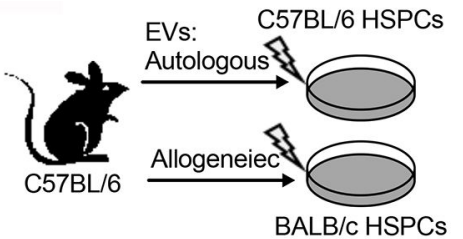

C

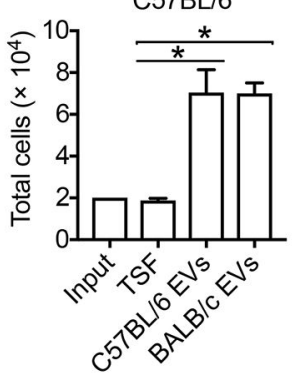

E

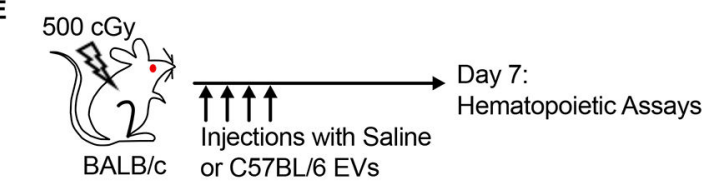

B

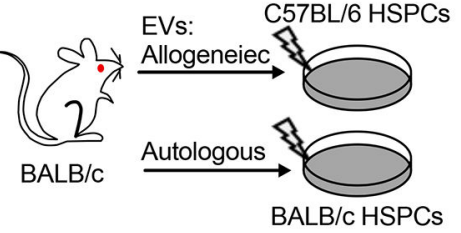

D

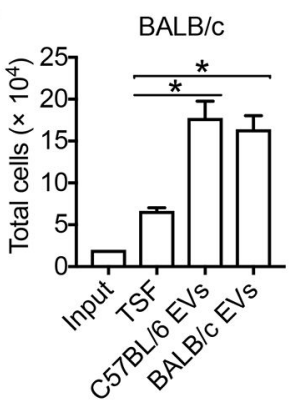

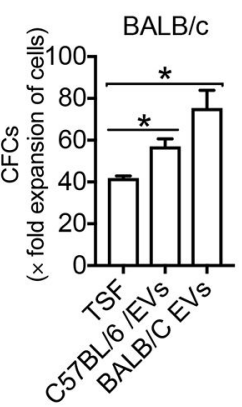

$\mathbf{F}$

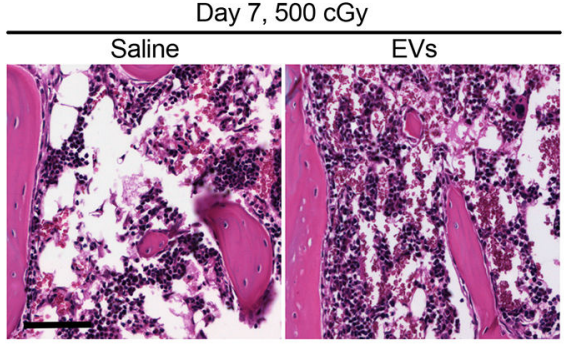

G

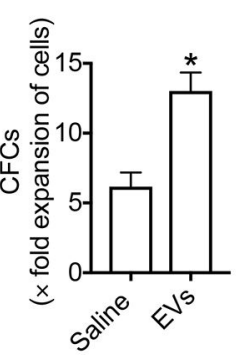

Figure 4. Autologous or allogeneic treatment of EVs yield comparable hematopoietic response following irradiation.

(A) Schematic diagram of study design. EVs from C57BL/6 marrow ECs were cultured with irradiated (300 cGy) BM Lin- cells from either C57BL/6 or BALB/c mice. (B) Schematic diagram of study design. EVs from BALB/c marrow ECs were cultured with irradiated (300 cGy) BM Lin- cells from either C57BL/6 or BALB/c mice. (C) Total cells and CFCs following 7-day culture of C57BL/6 Lin- cells with EVs generated from C57BL/6 ECs or BALB/c ECs. $n=3-5$ per group. * $p=0.001$ and $<0.0001$ for C57BL/6 EVs and BALB/c total cells compared to TSF, respectively. $* p<0.0001$ and 0.0006 for C57BL/6 EVs and BALB/c EVs CFCs compared to TSF, respectively. $p=0.2$ for C57BL/6 EVs vs BALB/c EVs. (D) Total cells and CFCs following 7-day culture of BALB/c Lin- cells with EVs generated from C57BL/6 ECs or BALB/c ECs. $n=3-6$ per group. * $p=0.0004$ and 0.0003 for $\mathrm{C} 57 \mathrm{BL} / 6 \mathrm{EVs}$ and BALB/c total cells compared to TSF, respectively. $* p=0.002$ and 0.003 for $\mathrm{C} 57 \mathrm{BL} / 6 \mathrm{EVs}$ and $\mathrm{BALB} / \mathrm{c} \mathrm{EVs}$ CFCs compared to TSF, respectively. $p=0.1$ for C57BL/6 EVs vs. BALB/c EVs. (E) Schematic diagram of study design. BALB/c mice were transplanted with C57BL/6 EVs or saline starting $24 \mathrm{~h}$ following $500 \mathrm{cGy}$-irradiation.

Hematopoietic assays were performed at day 7. (F) Hematoxylin and eosin-stained femurs at day 7 following 500 cGy. Scale bar $100 \mu \mathrm{m}$. (G) Levels of CFCs, $n=6$ per group. ${ }^{*} p=0.002$ for Saline vs. EVs 
A

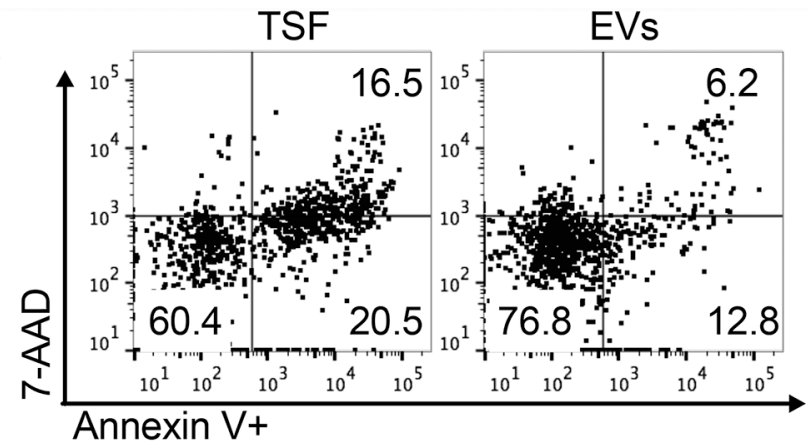

B
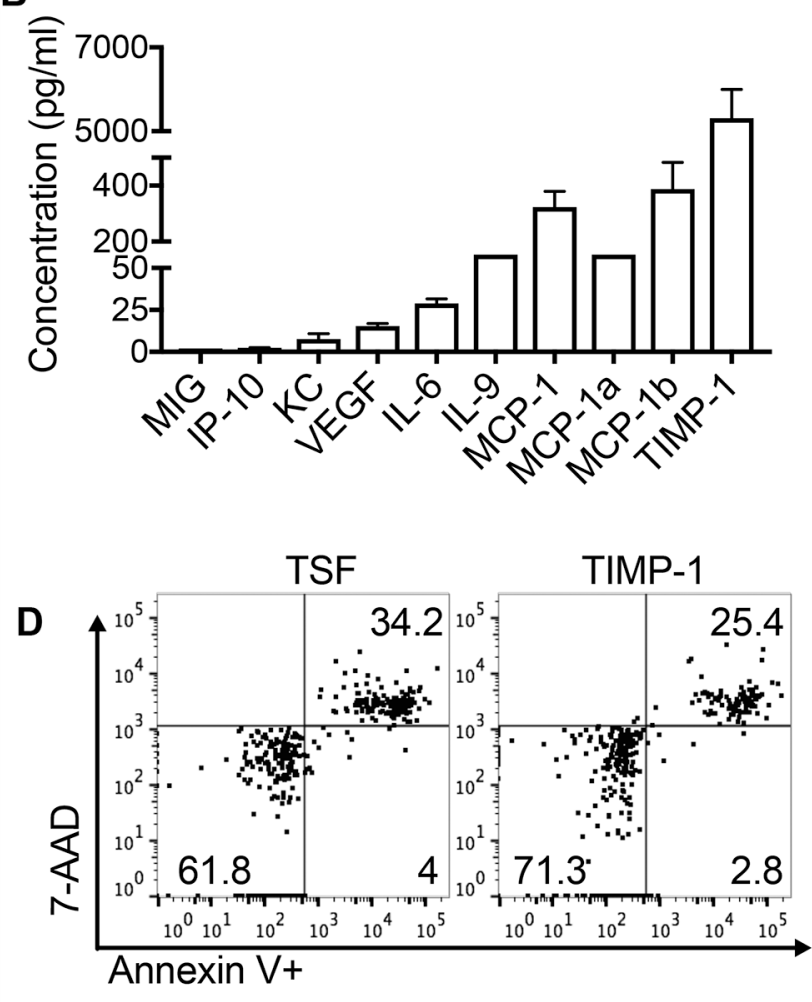

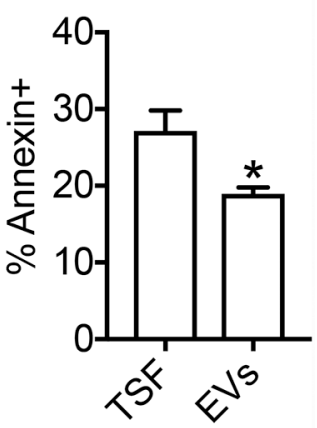

C
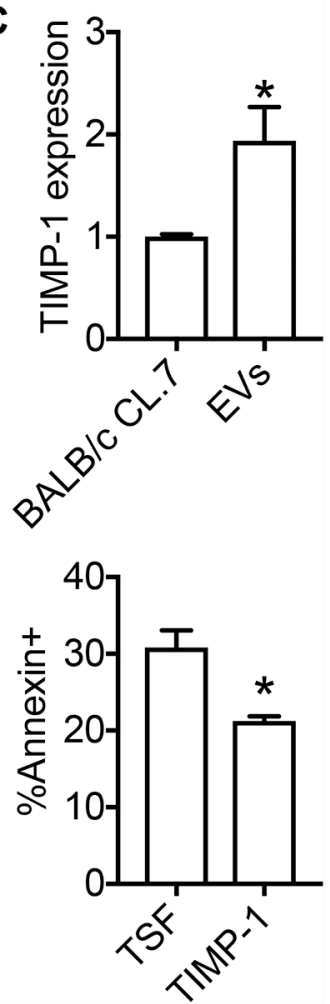

Figure 5. EVs abrogate apoptosis in part through TIMP-1.

(A) Flow cytometric analysis of Annexin+ cells of C57BL/6 KSL cells and progeny following $300 \mathrm{cGy}$ and 7 days in culture with EVs or TSF alone. $n=4-6$ per group. ${ }^{*} p=0.04$.

(B) EV samples were analyzed on Milliplex Map Mouse Cytokine/Chemokine Magnetic Bead Panel I and II. Samples were run in triplicate or duplicate. Highest-expressing cytokines and chemokines are shown, $n=5$ biologic samples. (C) TIMP-1 mRNA expression in BALB/c CL.7 fibroblast cell line, C57BL/6 EVs or C57BL/6 EC. $n=4-8$ per group. $* p=0.002$ and $<0.0001$ for EVs and EC compared to BALB/c CL.7, respectively. (D) Flow cytometric analysis of Annexin+ cells of C57BL/6 Lin- cells following $300 \mathrm{cGy}$ and $72 \mathrm{~h}$ in culture with TSF $+50 \mathrm{ng} / \mathrm{ml}$ TIMP- 1 or TSF alone. $n=4$ per group. ${ }^{*} p=0.006$. Data are shown as means \pm SEM. Student's $t$ test (two-tailed with unequal variance) were applied to these data. 